\title{
Determining Parameters of Simple Geometric Shaped Self-potential Anomalies
}

\author{
Maliheh Dehbashi ${ }^{1 *}$ and Mirsattar Meshinchi As/ ${ }^{2}$ \\ 'Student of Geophysics, Kermanshah Science and Research Branch, Islamic Azad University, Iran; \\ Mdjeofizik1@gmail.com \\ ${ }^{2}$ Departments of Geophysics, Kermanshah Science and Research Branch, Islamic Azad University, Iran
}

\begin{abstract}
A quantitative interpretation method of Self-Potential (SP) field data related to simple geometric models such as cylinders, spheres bodies has been used in this paper. The method is implemented for determining the center depth, electric dipole moment, polarization angle, and geometric shape of an underground buried body from field data related to simple geometric models. The method is based on mathematical modeling by using the least squares inversion. Some simple geometric shaped SP model has been formulated to describe the geophysical problem related to these buried simple geometric shaped models. This algorithm is chosen for being robust and also because its application to SP data converges rapidly towards the optimal estimation of parameters. To demonstrate the utility and validity of this method, three known theoretical models with a random noise of $2.5,5$, and $7.5 \%$ have been studied, where a very close agreement was obtained between assumed and calculated parameters of the models. This method has been applied on real field SP anomalies taken from Iran.
\end{abstract}

Keywords: Self-Potential Parameters, Self-Potential Interpretation, Least Squares Inversion.

\section{Introduction}

SP measurement is a geo-electric method used in many areas in geophysical exploration for mining, hydrologic geology, geothermal exploration, archaeology, mountain sliding, etc. In the recent years, it has been also used in some monitoring projects of earthquake prediction and volcanic eruption. The SP method is one of the oldest methods and enjoys wide applications in sulphides and graphite exploration and in geophysical groundwater investigations. The SP method has been most commonly used since 1931 for mineral exploration ${ }^{1,2}$. It has also been effectively used for water resource management by mapping the extension of water tables and hydrothermal systems. In hydro geological studies, SP is used to determine the distribution of saturated ground water and to determine the extent of contaminated ground water plumes $^{3}$. In petroleum and mineral exploration, SP is often used to characterize electrical bedrock properties in vertical borehole cross-sections. In geothermal exploration, SP can be used to rapidly locate hydrothermal areas, prior to applying more expensive and complex techniques such as ground resistivity and drilling. The SP method has a wide range of applications in Engineering and Geotechnical investigations ${ }^{4}$, Geothermal exploration $^{5-7}$ cavity detection ${ }^{8}$ and in the exploration for minerals, particularly metallic sulfides ${ }^{9}$.

The SP method is a passive geophysical method, in which natural spatial and temporal variations in the electrical potential field are measured on the surface of

*Author for correspondence: 
the earth or in boreholes. SP is an electrical method to measure the distribution of the natural electrical potential at the ground surface. It is the naturally occurring electrical potential of the earth resulting from geologic, geochemical, and hydrologic interactions which cause electric potentials to exist in the earth in the vicinity of the measurement point. With the recent development of new computer models, SP data can now be analyzed to acquire quantitative information on the depth and flow direction of the water or ore body generating the measured anomaly. The SP method consists in measuring the electrical potential at the surface of the Earth with respect to an electrode used as a reference.

SP is based on the variation of the natural electrical potential through the ground. The natural electrical current is generated by two main phenomena: electro kinetic and thermoelectric coupling 5 . However, other phenomena can also generate this current, such as rapid fluid disruption, ground resistivity contrasts, redox reactions, human activity, and the telluric field. In nature, the SP signal is generated by a complex interaction between each of these. The resulting SP maps and monitoring data are sensitive to flow processes in the subsurface ${ }^{10-12}$.

Interpretation of field measurements is challenging. First, many different phenomena (e.g. water fluxes in the vadose or saturated zone, gradients in chemical potential, or redox processes) can create SP signals and it is often unclear which source types will dominate the response at a given site. Second, accurate modeling of SP responses (to given source currents) can only be achieved when detailed knowledge about the electrical conductivity distribution is available. Third, the self potential method is a potential field method and the inverse problem of retrieving the source-current distribution in the subsurface is plagued by non-uniqueness. In any case, once corrected for anthropic signals and telluric currents, the SP signals evidence polarization phenomena occurring in the ground.

Sometimes an individual SP anomaly is found, that stands out so clearly that it can be separated from the regional background and the topographic interference, and is so simple in appearance that it can be modeled by a single polarized body. In this case, quantitative methods of interpretation can be used to determine the parameters of the polarized body by assuming a model with simple geometry. The model is considered realistic if the form and magnitude of calculated SP effects are close to the observed anomalies, and the model is geologically reasonable.

In this paper we review SP interpretation methods, followed by details of the formulation of the problem.

\section{SP Interpretation Methods}

One of the most important exploration problems is estimating the shape and depth of a buried structure. Different methods have been developed to determine the depth and the shape of the buried structure from SP data. The first category includes 2D and 3D continuous modeling and inversion methods ${ }^{13-15}$, which require current density and resistivity information as part of the input, along with some depth information obtained from geological and/or geophysical data. Thus, the resulting model can vary widely depending on these factors, but still give a calculated curve in close agreement with the observed data. The second category includes fixed simple geometry methods, in which the sphere, horizontal-cylinder, and vertical-cylinder models determine the depth and shape of the buried structure from residuals and/or observed SP data. The models may deviate from geological reality, but they are usually sufficient to determine whether the form and magnitude of the calculated SP effects are close enough to those observed to make the geological interpretation reasonable.

The SP anomaly expression produced by some polarized geologic structures can be represented by an approximately continuous function in shape (shape factor), depth, and polarization angle variables with an amplitude coefficient known as the electric dipole moment. The anomaly produced is typically associated only with particular sulfide or graphite ore deposits and generally is not related to other SP anomalies of interest, i.e., those related to fluid or heat flow. According to this simplified concept, different interpretation techniques are available in the literature for the quantitative interpretation of SP anomalies. These techniques are:

1. Methods were developed ${ }^{9,16-20}$ which used only a few points on the anomaly curve.

2. Curve Matching Techniques ${ }^{21}$, where the SP field curve is compared with sets of theoretical curves either manually or using a computer.

3. Least-squares methods. Here, the model parameters that give a best fit are derived using an initial presumption, by an iterative procedure ${ }^{22,23}$. 
4. Newly developed methods ${ }^{24}$ fall to the category of usage of derivative analysis and gradients.

5. Using a spectral analysis approach, in which the $S P$ data is analyzed and interpreted in the wave number domain by using the Fourier Transform (amplitude and phase spectra $)^{25}$.

We describe here a practical method of a Nonlinear Inversion $\mathrm{Technique}^{26}$ for interpreting SP anomalies due to simple geometrical structures using least squares method. Using the least squares method, a variant of linear equations has been solved in an attempt to estimate the geophysical parameters (e.g., electric dipole moment, depth and polarization angle) related to spheres, cylinders models. The method has been applied to synthetic data with and without random errors, and has been tested on field example from Iran.

\section{Formulation of the Problem}

The SP anomaly expressions produced by sphere-like, vertical cylinder-like, horizontal cylinder-like models, at any point on the free surface along the principal profile in a Cartesian Coordinate System are respectively given by equations $(1),(2),(3)^{19}$ :

$$
\begin{aligned}
& V\left(x_{i}\right)=k z^{2} \frac{x_{i} \cos \theta+z \sin \theta}{\left(x_{i}^{2}+z^{2}\right)^{3 / 2}} \quad(i=1,2, \ldots, N) \\
& V\left(x_{i}\right)=k \frac{x_{i} \cos \theta+z \sin \theta}{\left(x_{i}^{2}+z^{2}\right)^{1 / 2}} \quad(i=1,2, \ldots, N) \\
& V\left(x_{i}\right)=k z \frac{x_{i} \cos \theta+z \sin \theta}{x_{i}^{2}+z^{2}} \quad(i=1,2, \ldots, N)
\end{aligned}
$$

where,

$z$ is the depth from the surface to the center of the body,

$\theta$ is the polarization angle,

$k$ is the electric dipole moment (magnitude of polarization), and

$x_{i}(\mathrm{i}=1, \ldots, \mathrm{N})$ is the horizontal position coordinate.

After some simple algebra, equations (1), (2), (3) transform to equations (4), (5), (6) as follows:

$$
\begin{aligned}
& V_{i}^{2} x_{i}^{6}+3 V_{i}^{2} x_{i}^{2} z^{4}+3 V_{i}^{2} x_{i}^{4} z^{2}+V_{i}^{2} z^{6} \\
& =\left(k^{2} z^{4} x_{i}{ }^{2} \cos ^{2} \theta+k^{2} z^{6} \sin ^{2} \theta+k^{2} z^{5} x_{i} \sin 2 \theta\right) \\
& (i=1,2, \ldots, N)
\end{aligned}
$$

$$
\begin{aligned}
& V_{i}^{2} x_{i}^{2}+V_{i}^{2} z^{2}-k^{2} x_{i}^{2} \cos ^{2} \theta-k^{2} z^{2} \sin ^{2} \theta \\
& -k^{2} z x_{i} \sin 2 \theta=0 \quad(i=1,2, \ldots, N)
\end{aligned}
$$

$V_{i} x_{i}^{2}+V_{i} z^{2}-k z x_{i} \cos \theta-k z^{2} \sin \theta=0 \quad(i=1,2, \ldots, N)$

Equations (4), (5), (6) are nonlinear functions of the parameters $z, k$, and $\theta$. This nonlinearity is avoided by introducing new variables $q_{1}, q_{2}, q_{3}, q_{4}, q_{5}, q_{6}$ for Equation (4), $q_{7}, q_{8}, q_{9}, q_{10}$ for Equation (5), $q_{11}, q_{12}, q_{13}$ for Equation (6) which is defined as:

$$
\begin{aligned}
& q_{1}=z^{2}, q_{2}=z^{4}, q_{3}=z^{6}, q_{4}=k^{2} z^{4} \cos ^{2} \theta, \\
& q_{5}=k^{2} z^{6} \sin ^{2} \theta, q_{6}=k^{2} z^{5} \sin 2 \theta \\
& q_{7}=z^{2}, q_{8}=k^{2} \cos ^{2} \theta, q_{9}=k^{2} z^{2} \sin ^{2} \theta, \\
& q_{10}=k^{2} z \sin 2 \theta \\
& q_{11}=z^{2}, q_{12}=k z \cos \theta, q_{13}=k z^{2} \sin \theta
\end{aligned}
$$

Substituting equations (7), (8), (9) in equations (4), (5), (6) respectively, we obtain:

$$
\begin{gathered}
V_{i}^{2} x_{i}^{6}+3 V_{i}^{2} x_{i}^{4} q_{1}+3 V_{i}^{2} x_{i}^{2} q_{2} \\
+V_{i}^{2} q_{3}-x_{i}^{2} q_{4}-q_{5}-x_{i} q_{6}=0 \\
(i=1,2, \ldots, N) \\
V_{i}^{2} x_{i}^{2}+V_{i}^{2} q_{7}-x_{i}^{2} q_{8}-q_{9}-x_{i} q_{10}=0 \quad(i=1,2, \ldots, N) \\
V_{i} x_{i}^{2}+V_{i} q_{11}-x_{i} q_{12}-q_{13}=0 \quad(i=1,2, \ldots, N)
\end{gathered}
$$

Equations (10), (11), (12) are now linear functions of the variables $q_{i}(i=1, \ldots, 13)$.

\section{Least Squares Algorithm for the Inversion of SP Data}

The global optimal solutions of the linear system of equations (10), (11), (12) are obtained (in norm 2 in least squares sense) by minimizing the following mathematical objective functions respectively:

$$
\begin{aligned}
& \varphi_{1}(q)=\sum_{i=1}^{N}\left(\begin{array}{l}
V_{i}^{2} x_{i}^{6}+3 V_{i}^{2} x_{i}^{4} q_{1}+3 V_{i}^{2} x_{i}^{2} q_{2} \\
+V_{i}^{2} q_{3}-x_{i}^{2} q_{4}-q_{5}-x_{i} q_{6}
\end{array}\right)^{2} \\
& \varphi_{2}(q)=\sum_{i=1}^{N}\left(V_{i}^{2} x_{i}^{2}+V_{i}^{2} q_{7}-x_{i}^{2} q_{8}-q_{9}-x_{i} q_{10}\right)^{2}
\end{aligned}
$$




$$
\varphi_{3}(q)=\sum_{i=1}^{N}\left(V_{i} x_{i}^{2}+V_{i} q_{11}-x_{i} q_{12}-q_{13}\right)^{2}
$$

Equations (13), (14), (15) are easily solved by finding the unique solution of the following system of linear equations respectively:

$$
\begin{array}{cc}
\partial \varphi_{1}(q) / \partial q_{i}=0 & (i=1, \ldots, 6) \\
\left(\partial \varphi_{2}(q) / \partial q_{j}=0\right. & (j=7, \ldots, 10)) \\
\partial \varphi_{3}(q) / \partial q_{k}=0 & (k=11,12,13)
\end{array}
$$

These systems of simultaneous linear equations (16), (17), (18) could be written in matrix form respectively as:

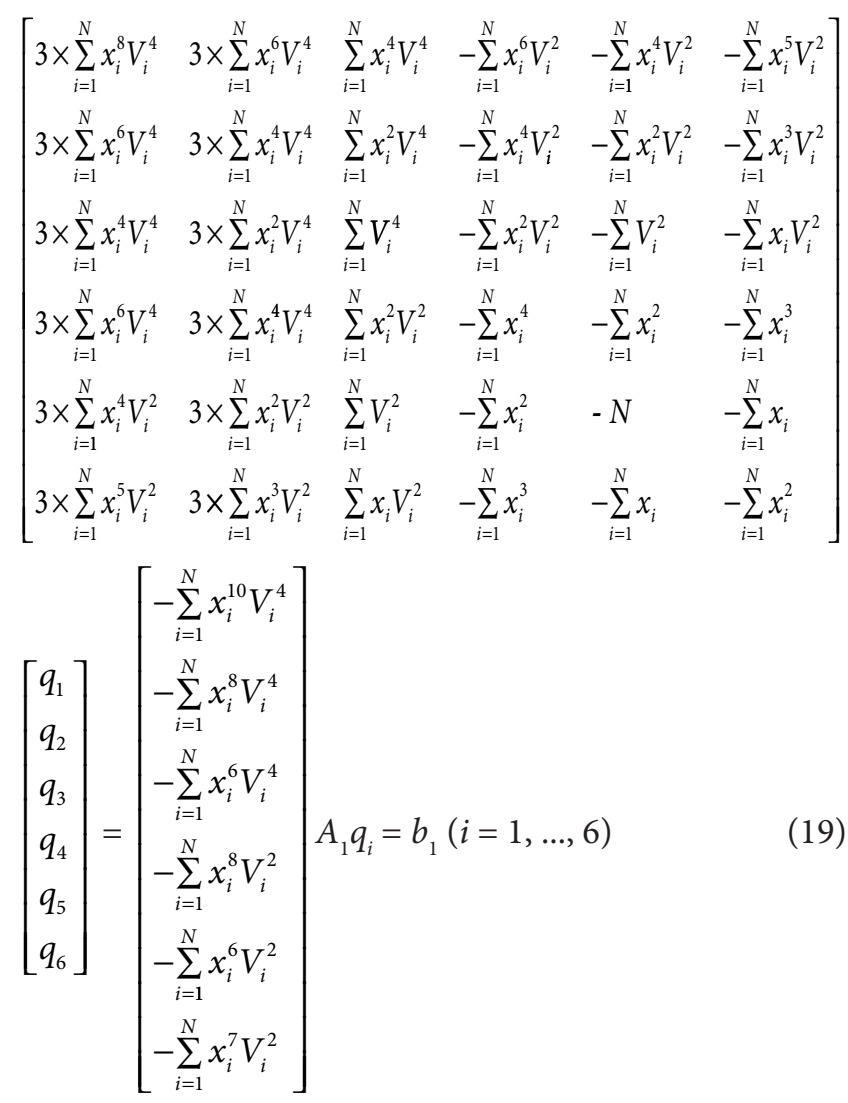

$$
\left[\begin{array}{llll}
-\sum_{i=1}^{N} V_{i}^{4} & \sum_{i=1}^{N} x_{i}^{2} V_{i}^{2} & \sum_{i=1}^{N} V_{i}^{2} & \sum_{i=1}^{N} x_{i} V_{i}^{2} \\
-\sum_{i=1}^{N} x_{i}^{2} V_{i}^{2} & \sum_{i=1}^{N} x_{i}^{4} & \sum_{i=1}^{N} x_{i}^{2} & \sum_{i=1}^{N} x_{i}^{3} \\
-\sum_{i=1}^{N} V_{i}^{2} & \sum_{i=1}^{N} x_{i}^{2} & N & \sum_{i=1}^{N} x_{i} \\
-\sum_{i=1}^{N} x_{i} V_{i}^{2} & \sum_{i=1}^{N} x_{i}^{3} & \sum_{i=1}^{N} x_{i} & \sum_{i=1}^{N} x_{i}^{2}
\end{array}\right]\left[\begin{array}{l}
q_{7} \\
q_{8} \\
q_{9} \\
q_{10}
\end{array}\right]=\left[\begin{array}{l}
\sum_{i=1}^{N} x_{i}^{2} V_{i}^{4} \\
\sum_{i=1}^{N} x_{i}^{4} V_{i}^{2} \\
\sum_{i=1}^{N} x_{i}^{2} V_{i}^{2} \\
\sum_{i=1}^{N} x_{i}^{3} V_{i}^{2}
\end{array}\right]
$$$$
A_{2} q_{j}=b_{2}(j=7, \ldots, 10)
$$

$$
\left[\begin{array}{ccc}
-\sum_{i=1}^{N} V_{i}^{2} & \sum_{i=1}^{N} x_{i} V_{i} & \sum_{i=1}^{N} V_{i} \\
-\sum_{i=1}^{N} x_{i} V_{i} & \sum_{i=1}^{N} x_{i}^{2} & \sum_{i=1}^{N} x_{i} \\
-\sum_{i=1}^{N} V_{i} & \sum_{i=1}^{N} x_{i} & N
\end{array}\right]\left[\begin{array}{l}
q_{11} \\
q_{12} \\
q_{13}
\end{array}\right]=\left[\begin{array}{l}
\sum_{i=1}^{N} x_{i}^{2} V_{i}^{2} \\
\sum_{i=1}^{N} x_{i}^{3} V_{i} \\
\sum_{i=1}^{N} x_{i}^{2} V_{i}
\end{array}\right]
$$

$A_{3} q_{k}=b_{3}(k=11,12,13)$

Since, $\mathrm{x}, \mathrm{V}$ vectors are known through $\mathrm{SP}$ data, it is clear that $A_{1}, A_{2}, A_{3}, b_{1}, b_{2}, b_{3}$ matrixes are also known, so the systems (19), (20), (21) have unique solutions $\mathrm{q}_{\mathrm{i}}$ $(\mathrm{i}=1, \ldots, 6), \quad \mathrm{q}_{\mathrm{j}}(\mathrm{j}=7, \ldots, 10), \quad \mathrm{q}_{\mathrm{k}}(\mathrm{k}=11,12,13)$ respectively. Once $\mathrm{q}_{\mathrm{i}}(\mathrm{i}=1, \ldots, 6), \quad \mathrm{q}_{\mathrm{j}}(\mathrm{j}=7, \ldots, 10), \quad \mathrm{q}_{\mathrm{k}}(\mathrm{k}=11,12,13)$ are calculated, one can obtain $\mathrm{z}$ (depth from the surface to the center of the body), $\theta$ (polarization angle, $\mathrm{k}$ (electric dipole moment) for sphere-like, vertical cylinder-like, horizontal cylinder-like models from equations (7), (8), (9), respectively. The signs of $\theta$ and $k$ are simply assigned by using the accordance between the field data anomaly and the computed one.

The above-mentioned procedure is essentially based on the statistical criteria of standard error $(\sigma)$. The smaller the $\sigma$, the closer $\mathrm{z}, \theta, \mathrm{k}$ to real $\mathrm{z}, \theta, \mathrm{k}$ of sphere-like, vertical cylinder-like, horizontal cylinder-like models. The smaller $\sigma$, the closer observed and evaluated values of SP anomaly curve. In other words, $\sigma$ is used in order to compare between the observed and evaluated values, and is defined as:

$$
\sigma=\sqrt{\frac{\sum_{i=1}^{N}\left(V_{i}(\text { observed })-V_{i}(\text { evaluated })\right)^{2}}{N}},
$$

where, $V_{i}$ (observed) and $V_{i}$ (evaluated) $(i=1, \ldots, N)$ are the observed and the evaluated values at the point $x_{i}$ $(i=1, \ldots, N)$, respectively.

$\sigma$ is also used to make a decision whether the causative body of SP anomaly is sphere-like, or vertical cylinder-like, or horizontal cylinder-like model. Decision procedure consists in choosing a model with minimum value of $\sigma$ between all models.

\section{Numerical Examples}

The described method is applied to both synthetic and real field data from Iran.

\subsection{Synthetic Data Example}

A Synthetic SP anomaly data set V (xi) $(\mathrm{i}=1, \ldots, \mathrm{N})$ due to a horizontal cylinder model for a profile length of 310 meters and arbitrary model parameters (the depth from the surface to the center of the body ( $\mathrm{z}=75$ meters), the 
electric dipole moment $(\mathrm{k}=100 \mathrm{mV}(0.1 \mathrm{~V}))$, the polarization angle ( $\theta=60$ degrees) is computed by applying equation (3). Thereafter, random white Gaussian noises of $2.5,5,7.5 \%$ were added to the above computed SP anomaly $\mathrm{V}(\mathrm{xi})(\mathrm{i}=1, \ldots, \mathrm{N})$. These four synthetic SP anomaly data sets are analyzed following the procedure of the described method in this paper, where the SP-estimated parameters are presented in Table 1.

Table 1. Clearly shows that the smallest values of the standard error in the four interpreted cases $\left(\sigma=6.2 \times 10^{-16}\right.$, $\left.\sigma=3 \times 10^{-4}, \sigma=0.01, \sigma=0.01\right)$ are obtained for a horizontal cylinder model. This indicates that the SP anomalies are preferably to be modeled as a horizontal cylinder-like structure. Modeling results presented in Table 1 also show a good agreement between assumed $(\mathrm{z}=75$ meters, $\mathrm{k}=0.1$ V, $\theta=60$ degrees) and estimated SP parameters $(\mathrm{z}=75,78$ meters, $\mathrm{k}=0.1,0.09 \mathrm{~V}, \theta=60,62$ degrees) in horizontal cylinder model, which consequently proves the high efficiency of the method.

\subsection{Field Data Example}

The procedure of the described method in this paper for the inversion of $S P$ data has been applied to interpret the SP anomaly obtained across a mineralized belt in Iran. The SP profiles (7 profiles in Figure 1) having the length of 310 meters has been digitized at an interval of 10 meters. The estimated parameters of each profile, obtained by the inversion of $S P$ data are shown in Table 2.

Table 1. Interpretation of a theoretical SP anomaly due to a horizontal cylinder model without noise with 2.5, $5,7.5 \%$ random white gaussian noises

\begin{tabular}{|c|c|c|c|c|c|}
\hline Assumed models & $\begin{array}{l}\text { Assumed SP } \\
\text { parameters }\end{array}$ & $\begin{array}{c}\text { Estimated SP } \\
\text { parameters } \\
\text { without noise }\end{array}$ & $\begin{array}{c}\text { Estimated SP } \\
\text { parameters } \\
\text { with } 2.5 \% \text { noise }\end{array}$ & $\begin{array}{c}\text { Estimated SP } \\
\text { parameters } \\
\text { with } 5 \% \text { noise }\end{array}$ & $\begin{array}{c}\text { Estimated SP } \\
\text { parameters } \\
\text { with } 7.5 \% \text { noise }\end{array}$ \\
\hline Sphere model & $\begin{aligned} \mathrm{z} & =75 \\
\mathrm{k} & =0.1 \\
\Theta & =60\end{aligned}$ & $\begin{aligned} \mathrm{z} & =134 \\
\mathrm{k} & =0.1 \\
\Theta & =25 \\
\sigma & =0.02\end{aligned}$ & $\begin{aligned} \mathrm{z} & =139 \\
\mathrm{k} & =0.1 \\
\Theta & =32 \\
\sigma & =0.03\end{aligned}$ & $\begin{aligned} \mathrm{z} & =134 \\
\mathrm{k} & =0.1 \\
\Theta & =46 \\
\sigma & =0.05\end{aligned}$ & $\begin{aligned} z & =151 \\
k & =0.03 \\
\Theta & =69 \\
\sigma & =0.06\end{aligned}$ \\
\hline $\begin{array}{l}\text { Vertical cylinder } \\
\text { model }\end{array}$ & $\begin{aligned} z & =75 \\
k & =0.1 \\
\Theta & =60\end{aligned}$ & $\begin{aligned} \mathrm{z} & =44 \\
\mathrm{k} & =0.09 \\
\Theta & =76 \\
\sigma & =0.01\end{aligned}$ & $\begin{aligned} \mathrm{z} & =40 \\
\mathrm{k} & =0.1 \\
\Theta & =76 \\
\sigma & =0.01\end{aligned}$ & $\begin{aligned} \mathrm{z} & =69 \\
\mathrm{k} & =0.1 \\
\Theta & =68 \\
\sigma & =0.04\end{aligned}$ & $\begin{aligned} \mathrm{z} & =101 \\
\mathrm{k} & =0.1 \\
\Theta & =63 \\
\sigma & =0.05\end{aligned}$ \\
\hline $\begin{array}{l}\text { Horizontal } \\
\text { cylinder } \\
\text { model }\end{array}$ & $\begin{aligned} \mathrm{z} & =75 \\
\mathrm{k} & =0.1 \\
\Theta & =60\end{aligned}$ & $\begin{aligned} \mathrm{z} & =75 \\
\mathrm{k} & =0.1 \\
\Theta & =60 \\
\sigma & =6.2 \times 10^{-16}\end{aligned}$ & $\begin{aligned} \mathrm{z} & =78 \\
\mathrm{k} & =0.09 \\
\Theta & =62 \\
\sigma & =3 \times 10^{-4}\end{aligned}$ & $\begin{aligned} \mathrm{z} & =85 \\
\mathrm{k} & =0.1 \\
\Theta & =46 \\
\sigma & =0.01\end{aligned}$ & $\begin{aligned} \mathrm{z} & =91 \\
\mathrm{k} & =0.1 \\
\Theta & =47 \\
\sigma & =0.01\end{aligned}$ \\
\hline
\end{tabular}

Table 2. Estimated SP parameters (depth from the surface to the center of the body z, electric dipole moment $\mathrm{k}$, polarization angle $\Theta$, standard error $\sigma$ ) and preferable synthetic models which is closest to the observed SP curves in profiles 1-7 in Iran (see Figure 1)

\begin{tabular}{|c|c|c|c|c|c|}
\hline $\begin{array}{l}\text { Profile } \\
\text { No. }\end{array}$ & $\begin{array}{l}\text { Preferable Synthetic } \\
\text { Model }\end{array}$ & $\begin{array}{l}\text { Depth from the surface } \\
\text { to the center of the } \\
\text { body }(\mathrm{z})(\text { Meter })\end{array}$ & $\begin{array}{c}\text { Electric dipole } \\
\text { moment }(\mathrm{k}) \\
(\mathrm{mV})\end{array}$ & $\begin{array}{c}\text { Polarization } \\
\text { angle }(\Theta) \\
(\text { Degree })\end{array}$ & $\begin{array}{l}\text { Standard } \\
\text { Error } \\
(\sigma)\end{array}$ \\
\hline 1 & $\begin{array}{l}\text { Vertical cylinder } \\
\text { model with } 5 \% \text { noise }\end{array}$ & 181 & 19 & -13 & 3.7 \\
\hline 2 & $\begin{array}{l}\text { Vertical cylinder } \\
\text { model with } 5 \% \text { noise }\end{array}$ & 88 & 13 & 62 & 2.6 \\
\hline 3 & $\begin{array}{l}\text { Sphere model with 5\% } \\
\text { noise }\end{array}$ & 145 & 20 & 48 & 6.4 \\
\hline 4 & $\begin{array}{l}\text { Sphere model with } 7.5 \% \\
\text { noise }\end{array}$ & 199 & 9 & 35 & 5.3 \\
\hline 5 & $\begin{array}{l}\text { Horizontal cylinder } \\
\text { model with } 7.5 \% \text { noise }\end{array}$ & 114 & -7 & -55 & 2.8 \\
\hline 6 & $\begin{array}{l}\text { Horizontal cylinder } \\
\text { model with } 7.5 \% \text { noise }\end{array}$ & 117 & 7 & 43 & 3.7 \\
\hline 7 & $\begin{array}{l}\text { Vertical cylinder } \\
\text { model with } 5 \% \text { noise }\end{array}$ & 222 & -17 & -12 & 2.9 \\
\hline
\end{tabular}



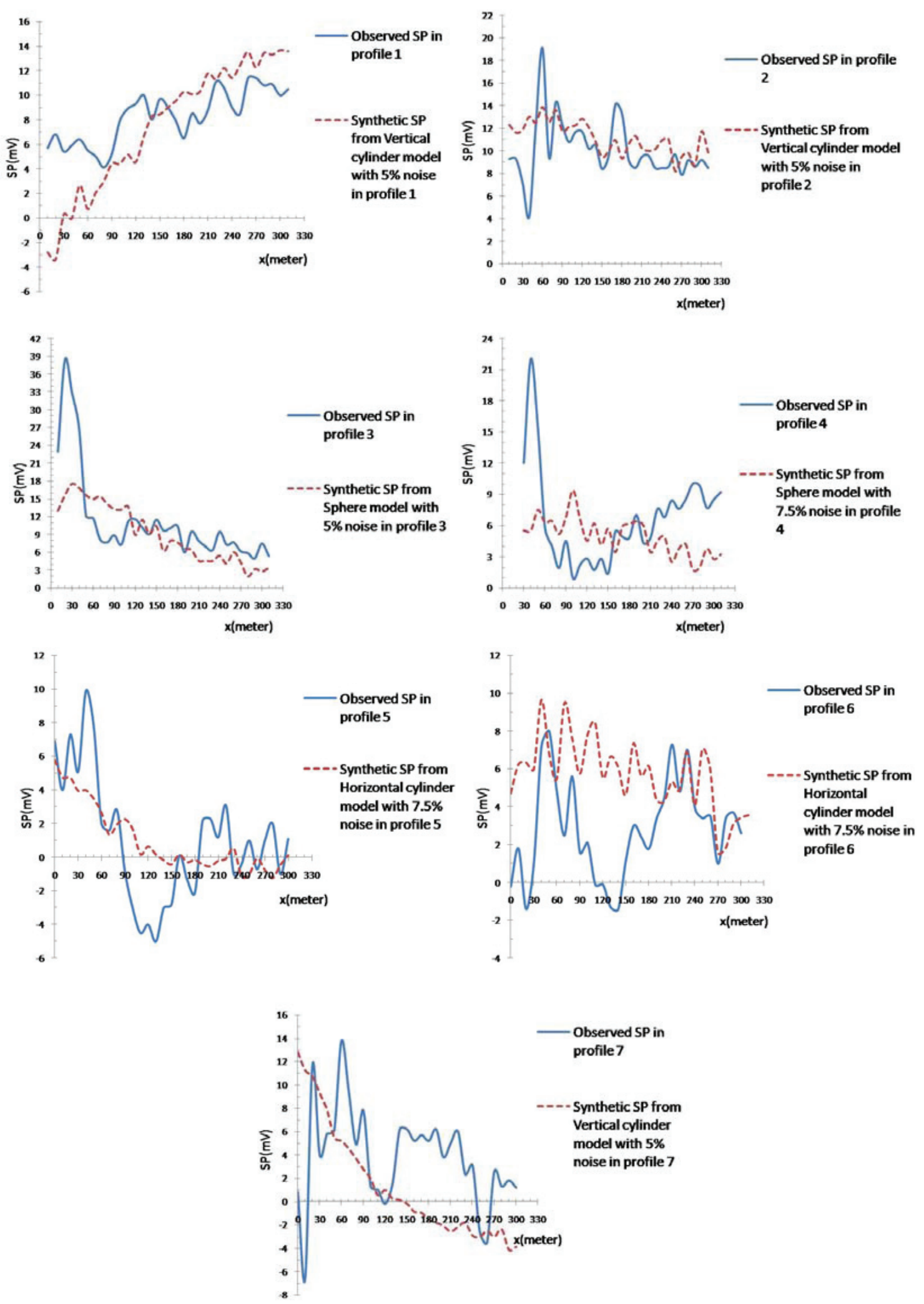

Figure 1. Comparison of observed and synthetic SP in profiles 1-7 in Iran. 


\section{Discussion and Conclusions}

In this paper, a new geophysical method is being used for the interpretation of $S P$ anomalies. The method is based on the least squares concept. First, a nonlinear model has been formulated to describe the geophysical problem related to the buried sphere and cylinder structures. This nonlinear geophysical model has been linearized by transforming model parameters. Then least squares technique is applied to linear model. The utilization of the least squares technique easily leads to the estimation of the SP parameters (Depth from the surface to the center of the body z, Electric dipole moment $k$, Polarization angle $\theta$ ). The advantages of the least squares technique are: 1) all observed values are used during the interpretation process. 2) The technique is automatic. The proposed approach has been validated with synthetic and real data. The application of the least squares technique to a set of practical field data resulted in good agreement between observed and computed data. Therefore, the proposed technique can be used for routine analysis of SP data to determine the parameters with ease and accuracy.

\section{Acknowledgement}

I take this opportunity to express my profound gratitude to all who have supported me in my research work.

\section{References}

1. Ewing S. The copper-copper sulfate half-cell for measuring potentials in earth. American Gas Association Proceedings; 1939; 21:624-34.

2. Poldini E. Geophysical exploration by spontaneous polarization methods. Mining magazine. 1939; 60: 22-7, 90-4.

3. Naudet V, Revil A, Rizzo E. Bottero J-Y, Beggasat P. Groundwater redox conditions and conductivity in a contaminant plume from geoelectrical investigations. Hydrol Earth Syst Sc. 2004; 8(1):8-22.

4. Markiewicz RD, Davenport GC, Randall JA. The use of self potential surveys in geotechnical investigations. 54th Annual International Meeting Society of Exploration Geophysicists, Expanded Abstracts. 1984 Dec 2-6; Atlanta, Georgia: Society of Exploration Geophysics.

5. Corwin RF, Hoover DB. The self-potential method in geothermal exploration. Geophysics. 1979; 44(2):226-45.

6. Fitterman DV, Corwin RF. Inversion of self-potential data from the Cerro-Prieto geothermal field Mexico. Geophysics. 1982; 47(6):938-45.
7. Anderson LA. Self-potential investigations in the Puhimau thermal area, Kilauea Volcano, Hawaii. 54th Annual International Meeting, Society of Exploration Geophysicists, Expanded Abstracts. 1984; Society Exploration Geophysicists. 1:84-6.

8. Schiavone D, Quarto R. Cavities detection using the self potential method. 54th Meeting, European Association Society Exploration Geophysicists. Abstracts of the European Association Exploration Geophysicists; 1992:362-63.

9. Yungul S. Interpretation of spontaneous polarization anomalies caused by spheroidal ore bodies. Geophysics. 1950; 15(2):237-46.

10. Doussan C, Jouniaux L, Thony J-L. Variations of self potential and unsaturated water flow with time in sandy loam and clay loam soils. J Hydrol. 2002; 267(3):173-85.

11. Rizzo E, Suski B, Revil A, Straface S, Troisi S. Selfpotential signals associated with pumping tests experiments. J Geophys Res. 2004; 109(B10):203. doi: 10.1029/2004JB003049.

12. Suski B, Revil A, Titov K, Konosavsky P, Voltz M, Dagès C, Huttel O. Monitoring of an infiltration experiment using the self-potential method. Water Resour Res. 2006; 42(8):W08418. doi: 10.1029/2005WR004840.

13. Guptasarma D. Effect of surface polarization on resistivity modeling. Geophysics. 1983; 48(1):98-106.

14. Furness P. Modelling spontaneous mineralization potentials with a new integral equation. J Appl Geophys. 1992; 29(2):143-55.

15. Shi W, Morgan FD. Non-uniqueness in self-potential inversion. 66th Annual International Meeting; 1996. Society of Exploration Geophysicists. Extended Abstracts, p. 950-3.

16. DeWitte L. A new method of interpretation of self-potential data. Geophysics. 1948; 13(4):600-8.

17. Paul MK. Direct interpretation of self-potential anomalies caused by inclined sheets of infinite horizontal extensions. Geophysics. 1965; 30(3):418-23.

18. Paul MK, Datta S, Banerjee B. Interpretation of self potential anomalies due to localized causative bodies. Pure Appl Geophys. 1965; 61(6):95-100.

19. Bhattacharya BB, Roy N. A note on the use of nomograms for self-potential anomalies. Geophys Prospect. 1981; 29(1):102-07.

20. Rao AD, Ram Babu HV. Quantitative interpretation of self potential anomalies due to two-dimensional sheet-like bodies. Geophysics. 1983; 48(12):1659-64.

21. Meiser P. A method of quantitative interpretation of self-potential measurements. Geophys Prospect. 1962; 10(2):203-18.

22. Abdelrahman EM, El-Araby TM, Ammar AA, Hassanein HI. A least-squares approach to shape determination from self-potential anomalies. Pure Appl Geophys. 1997; 150(1):121-28. 
23. Abdelrahman EM, Sharafeldin SM. A least-squares approach to depth determination from residual selfpotential anomalies caused by horizontal cylinders and spheres. Geophysics. 1997; 62(1):44-8.

24. Abdelrahman EM, Ammar AA, Hassanein HI, Hafez MA. Derivative analysis of SP anomalies. Geophysics. 1998; 63(3):890-497.

25. Rao AD, Ram Babu HV, Sivakumar Sinha GDJ. A fourier transform method for the interpretation of self-potential anomalies due to two to two-dimensional inclined sheets of finite depth extent, Pure Appl Geophys. 1982; 120(2): 365-374.

26. Tlas M, Asfahani J. Using of the Adaptive Simulated Annealing (ASA) for quantitative interpretation of selfpotential anomalies due to simple geometrical structures. Journal of King Abdulaziz Univeristy (JKAU): Earth Sciences. 2007; 19(1):99-118. 